\title{
ON EXTREMAL QUASI-MODULAR FORMS AFTER KANEKO AND KOIKE
}

\author{
Federico PELLARIN \\ With an appendix by Gabriele NEBE
}

(Received 19 October 2019)

\begin{abstract}
Kaneko and Koike introduced the notion of extremal quasi-modular forms and proposed conjectures on their arithmetic properties. The aim of this paper is to prove a rather sharp multiplicity estimate for these quasi-modular forms. The paper ends with discussions and partial answers around these conjectures and an appendix by G. Nebe containing the proof of the integrality of the Fourier coefficients of the normalized extremal quasi-modular form of weight 14 and depth one.
\end{abstract}

\section{Introduction}

In [5], Kaneko and Koike introduced the notion of extremal quasi-modular forms and discussed the potential arithmetic interest of these functions. Let $E_{2}(z), E_{4}(z)$ and $E_{6}(z)$ be the Eisenstein series of weights two, four and six, respectively, normalized to have limit one as the imaginary part $\Im(z)$ of $z$, the variable in the complex upper half-plane $\mathcal{H}$, tends to infinity. These functions have quite explicit series expansions in $\mathbb{Z}[[q]]$ in terms of the uniformizer $q=e^{2 \pi i z}$, convergent for $|q|<1$ :

$$
\begin{gathered}
E_{2}(z)=1-24 \sum_{n \geq 1} \sigma_{1}(n) q^{n}, \quad E_{4}(z)=1+240 \sum_{n \geq 1} \sigma_{3}(n) q^{n}, \\
E_{6}(z)=1-504 \sum_{n \geq 1} \sigma_{5}(n) q^{n},
\end{gathered}
$$

where $\sigma_{k}(n)$ denotes the sum $\sum_{d \mid n} d^{k}$ over the positive divisors of $n$. It is well known and easy to prove that these functions are algebraically independent over $\mathbb{C}$ and the threedimensional polynomial algebra $\tilde{M}:=\mathbb{C}\left[E_{2}, E_{4}, E_{6}\right]$ is graded by the respective weights two, four and six of $E_{2}, E_{4}$ and $E_{6}$. A quasi-modular form of weight $w$ is a polynomial in $E_{2}, E_{4}$ and $E_{6}$ homogeneous of weight $w$. In particular, such a function $f$, when non-zero, can be written in a unique way as

$$
f=f_{0}+f_{1} E_{2}+\cdots+f_{l} E_{2}^{l}
$$

with $f_{i}$ a modular form of weight $w-2 i$ for all $i$ and $f_{l} \neq 0$. We refer to the integer $l$ as the depth of $f$. Let $\widetilde{M}_{w}^{\leq l}$ be the finite-dimensional vector space of quasi-modular forms of weight

2010 Mathematics Subject Classification: Primary 11F11, 11H56.

Keywords: extremal quasimodular forms; multiplicity estimates; Leech lattice and automorphisms.

(C) 2020 Faculty of Mathematics, Kyushu University 
$w \in 2 \mathbb{Z}$ and depth $\leq l$. We have

$$
\widetilde{M}=\mathbb{C}\left[E_{2}, E_{4}, E_{6}\right]=\bigcup_{l \geq 0} \bigoplus_{w} \tilde{M}_{w}^{\leq l} .
$$

By (1) the $\mathbb{C}$-algebra $\tilde{M}$ embeds in the subring of series of $\mathbb{C}[[q]]$ that are converging for $|q|<1$. From now on, we identify quasi-modular forms with the formal series representing their $q$-expansions (Fourier series expansions). If $f \in \mathbb{C}[[q]]$, we set

$$
v(f):=\operatorname{ord}_{q=0}(f) \in \mathbb{N} \cup\{\infty\},
$$

where $\mathbb{N}=\{n \in \mathbb{Z}: n \geq 0\}$. This is the order of vanishing at $q=0$ of the Fourier series of $f$ and defines a valuation over the $\mathbb{C}$-algebra $\tilde{M}$.

The dimension $\delta_{l}(w)$ of $\tilde{M}_{w}^{\leq l}$ can be computed in the following way:

$$
\delta_{l}(w)=\sum_{i=0}^{l} d(w-2 i),
$$

where $d(w)$ denotes, for $w \in 2 \mathbb{Z}$, the dimension of the $\mathbb{C}$-vector space $M_{w}$ of modular forms of weight $w$ (which is also equal to $\widetilde{M}_{w}^{\leq 0}$ ). We recall that

$$
d(w)= \begin{cases}0 & \text { if } w<0, \\ \lfloor w / 12\rfloor & \text { if } w \geq 0 \text { and } w \equiv 2(\bmod 12), \\ \lfloor w / 12\rfloor+1 & \text { in all other cases. }\end{cases}
$$

If $w-2 l=2$ with $w$ even, $\tilde{M}_{w}^{\leq l}$ is non-zero but there are no quasi-modular forms of weight $w$ and depth $l$ because $d(2)=0$.

The following definition is due to Kaneko and Koike [5].

Definition 1.1. A quasi-modular form $f$ of weight $w$ and depth $l$ is extremal if

$$
v(f)=\delta_{l}(w)-1 .
$$

In [5] Kaneko and Koike address the following question.

Does there always exist an extremal quasi-modular form of given weight $w$ and depth $l$, provided $w$ and $l$ satisfy the necessary constraint $0 \leq 2 l \leq w$, $2 l \neq w-2$ ? And is it unique when normalized?

We cannot answer this question in full generality, but in the present paper we show that the answer is affirmative if we suppose that $l \leq 4$.

Assume that $\tilde{M}_{w}^{\leq l} \neq\{0\}$. We set:

$v_{\max }(l, w):=\max \left\{n \in \mathbb{N}\right.$ such that there exists $f \in \tilde{M}_{w}^{\leq l} \backslash\{0\}$ with $\left.v(f)=n\right\} \in \mathbb{N}$.

Note that

$$
v_{\max }(l, w) \geq \delta_{l}(w)-1 .
$$

To see this we set $\delta=\delta_{l}(w)$ and we consider a basis $\left(g_{0}, \ldots, g_{\delta-1}\right)$ of $\tilde{M}_{w}^{\leq l}$. Writing the $q$-expansion of each element of the basis $g_{i}=\sum_{j \geq 0} g_{i, j} q^{j} \in \mathbb{C}[[q]]$, the matrix

$$
\left(g_{i, j}\right)_{\substack{0 \leq i \leq \delta-1 \\ 0 \leq j \leq \delta-2}}
$$

has rank $\leq \delta-1$ which justifies (3). 
Definition 1.2. A quasi-modular form $f$ of weight $w$ and depth $\leq l$ is analytically extremal if

$$
v(f)=v_{\max }(l, w) .
$$

For any $l, w$ such that $\widetilde{M}_{\mathfrak{w}}^{\leq l} \neq\{0\}$ there exists a unique normalized analytically extremal quasi-modular form $f_{l, w} \in \widetilde{M}_{w}^{\leq l} \backslash\{0\}$. We may reinforce the terminology by saying that an extremal form in the sense of Definition 1.1 is algebraically extremal. The attribute 'algebraic' is chosen because the definition seems to rather involve the algebraic structure of the $\mathbb{C}$-vector space $\tilde{M}_{w}^{\leq l}$.

THEOREM 1.3. Let $l$ be in the set $\{0,1,2,3,4\}$ and assume that $0 \leq 2 l \leq w v 2 l \neq w-2$. Then a non-zero quasi-modular form of weight $w$ and depth $l$ is algebraically extremal if and only if it is analytically extremal.

This is a direct consequence of Theorem 2.3 below and answers Kaneko and Koike's question from above, in the case $l \leq 4$. For $l$ in this range, Definitions 1.1 and 1.2 therefore coincide and there is no need to specify 'algebraically' or 'analytically' as an attribute for extremality.

In [5], Kaneko and Koike ask whether an element in $\tilde{M}_{k}^{\leq l}$ is uniquely determined by its first $\delta_{l}(k)$ Fourier coefficients and if one can prescribe these coefficients arbitrarily. In other words, they ask if there exists a basis of $\widetilde{M}_{k}^{\leq l}$ the $q$-expansions of the elements of which agree with the canonical diagonals basis $\left(1, q, q^{2}, \ldots\right)$ of the vector space $\mathbb{C}[[q]]$ on its first $\delta_{l}(w)$ elements (we may call this a 'diagonal basis' of $\widetilde{M}_{k}^{\leq l}$ ). For $l=0$ we have the so-called Miller bases, which answer positively this question; they can be easily constructed by the fact that the algebra of modular forms has dimension two. For higher depth we do not know a general answer to the question but Theorem 2.3 implies that such bases exist for $l \leq 4$. Indeed, completing the matrix in (4) by adding a column, $U=\left(f_{i, j}\right)_{0 \leq i, j \leq \delta-1}$ is nonsingular, because otherwise we would be able to construct, by linear combination, a non-zero element $f \in \widetilde{M}_{k}^{\leq l} \backslash\{0\}$ such that $v(f)>v_{\max }(l, w)=\delta_{l}(w)-1$, which is impossible (the last equality follows from Theorem 1.3). Hence 'diagonal bases' for $\widetilde{M}_{k}^{\leq l}, l \leq 4$, exist.

\section{A multiplicity estimate}

How large can $v_{\max }(l, m)$ be? Classically, a simple multiplicity estimate holds on $f \in \widetilde{M}_{w}^{\leq l}$. For example, it is easy to show, by using an elementary resultant argument, that

$$
v_{\max }(l, w) \leq 3 \delta_{l}(w) .
$$

The main result of this section is Theorem 2.3, where we prove a rather sharp multiplicity estimate for quasi-modular forms. For $w \in \mathbb{Z}$ we consider the difference

$$
\kappa_{l}(w):=d((l+1) w)-\delta_{l}(w)=\operatorname{dim}_{\mathbb{C}}\left(M_{(l+1) w}\right)-\operatorname{dim}_{\mathbb{C}}\left(\tilde{M}_{w}^{\leq l}\right) \in \mathbb{Z} .
$$

We have the following elementary lemma.

LEMMA 2.1. For all $l \geq 0$ the sequence $\left(\kappa_{l}(w)\right)_{w \geq 0,2 \mid w}$ is non-negative, increasing and there exists an integer $0 \leq \kappa_{l} \leq 6^{-1}(3+l)(4+l)$ such that for all $w \geq 2 l+12, \kappa_{l}(w)=\kappa_{l}$. Moreover, $\kappa_{l}(w)=\kappa_{l}=0$ for all $w \in 2 \mathbb{Z}$ if $l \in\{0,1,2,3,4\}$. 
Proof. We note that if $w>12$ then $d(w)=1+d(w-12)$. Hence, if $w \geq 2 l+12$ we can write $w=w^{\prime}+12$ with $w^{\prime}-2 i \geq 0$ for all $i \leq l$ and

$$
\kappa_{l}(w)=l+1+d\left((l+1) w^{\prime}\right)-(l+1)-\sum_{i=0}^{l} d\left(w^{\prime}-2 i\right)=\kappa_{l}\left(w^{\prime}\right) .
$$

From this computation we also see that the sequence is increasing. Moreover, since $d(w) \leq$ $w / 12+1$ for all $w \geq 0$ by (2), we have the trivial upper bound

$$
\kappa_{l}(w) \leq \frac{(l+1)(2 l+12)}{12}+1
$$

if $w \geq 0$, which yields the one for $\kappa_{l}$. Finally, the fact that $\kappa_{l}(w)=0$ for all $w \geq 0$ and for all $l \in\{0,1,2,3,4\}$ is trivial for $l=0$ and otherwise follows from the following identities, valid for any $w \in 2 \mathbb{Z}$ :

$$
\begin{aligned}
& d(2 w)=d(w)+d(w-2), \\
& d(3 w)=d(w)+d(w-2)+d(w-4), \\
& d(4 w)=d(w)+d(w-2)+d(w-4)+d(w-6), \\
& d(5 w)=d(w)+d(w-2)+d(w-4)+d(w-6)+d(w-8),
\end{aligned}
$$

which can be proved with elementary computations using (2) and are left to the reader.

Remark 2.2. The first coefficients of the sequence $\kappa_{l}$ are

$$
0,0,0,0,0,1,1,2,3,4,5,7,8,10,12,14,16,19, \ldots
$$

Let $a(n)$ be the cardinality of the set $\left\{(i, j, k) \in \mathbb{N}^{3}: i+2 j+3 k=n\right\}$ (set to zero if $n \leq 0$ ). Then, the first coefficients of the sequence $\kappa_{l}$ agree with the first coefficients of the sequence $a(n-6)$. Numerical experiments suggest that

$$
\kappa_{l}(w)=a(l)-a(l-w / 2), \quad \forall l \geq 0, \quad w \in 2 \mathbb{Z} .
$$

THEOREM 2.3. (Multiplicity estimate) The following inequality holds:

$$
v_{\max }(l, w) \leq \delta_{l}(w)-1+\kappa_{l} .
$$

Proof. For

$$
\gamma=\left(\begin{array}{ll}
a & b \\
c & d
\end{array}\right) \in \Gamma:=\mathrm{SL}_{2}(\mathbb{Z})
$$

and $z \in \mathcal{H}$ the complex upper half-plane, we write

$$
J_{\gamma}(z)=c z+d \quad \text { and } \quad L_{\gamma}(z)=\frac{c}{c z+d} .
$$

We will use the identity map $\rho_{1}: \mathrm{SL}_{2}(\mathbb{Z}) \rightarrow \mathrm{SL}_{2}(\mathbb{Z})$ so that $\rho_{1}(\gamma)=\gamma$, and its symmetric powers of order $l \geq 1$,

$$
\rho_{l}=S^{l}\left(\rho_{1}\right): \mathrm{SL}_{2}(\mathbb{Z}) \rightarrow \mathrm{SL}_{l+1}(\mathbb{Z})
$$


realized in the space of polynomials homogeneous of degree $s=l+1$ with coefficients in $\mathbb{C}$ :

$$
\rho_{l}\left(\left(\begin{array}{ll}
a & b \\
c & d
\end{array}\right)\right)\left(X^{s-r} Y^{r}\right)=(a X+c Y)^{s-r}(b X+d Y)^{r} .
$$

For example, for $\gamma$ as above, in the basis $\left(X^{2}, X Y, Y^{2}\right)$ :

$$
\rho_{2}(\gamma)=\left(\begin{array}{ccc}
a^{2} & a b & b^{2} \\
2 a c & a d+b c & 2 b d \\
c^{2} & c d & d^{2}
\end{array}\right) .
$$

Let us also consider the derivation $D$ of $\mathbb{C}((q))$ induced by

$$
D=(2 \pi i)^{-1} \frac{d}{d z}=q \frac{d}{d q} .
$$

Then we have Ramanujan's differential system:

$$
D\left(E_{2}\right)=\frac{1}{12}\left(E_{2}^{2}-E_{4}\right), \quad D\left(E_{4}\right)=\frac{1}{3}\left(E_{2} E_{4}-E_{6}\right), \quad D\left(E_{6}\right)=\frac{1}{2}\left(E_{2} E_{6}-E_{4}^{2}\right) .
$$

Let $f \in \widetilde{M}_{w}^{\leq l}$ be a quasi-modular form of weight $w$ and depth $\leq l$. There exists, uniquely determined, a polynomial

$$
P_{f} \in \operatorname{Hol}(\mathcal{H})[X]
$$

of degree equal to the depth of $f$ with coefficients that are holomorphic functions $\mathcal{H} \rightarrow \mathbb{C}$, such that, for all $\gamma \in \Gamma$ and for all $z \in \mathcal{H}$, we have the functional equation

$$
f(\gamma(z))=J_{\gamma}(z)^{w} P_{f}\left(L_{\gamma}(z)\right) .
$$

It is plain that the coefficients of $P_{f}$ are quasi-modular forms. In particular, we can identify $P_{f}$ with an element of $\mathbb{C}[[q]][X]$. Note that, additionally, the constant term of $P_{f}$ is equal to $f$. We write $Q_{f}(x)=x^{l} P_{f}(1 / x) \in \mathbb{C}[[q]][x]$ and we set

$$
F_{f}(z)=\left(\begin{array}{c}
Q_{f} \\
\partial Q_{f} / \partial x \\
\partial^{2} Q_{f} / \partial x^{2} \\
\partial^{3} Q_{f} / \partial x^{3} \\
\vdots \\
\partial^{l} Q_{f} / \partial x^{l}
\end{array}\right)_{x=z}
$$

This defines a (weak) vectorial modular form of weight $w-l$ associated to $\rho_{l}$. In other words, the above is a holomorphic function $\mathcal{H} \rightarrow \mathbb{C}^{l}$ (column) which satisfies the following property: for all $\gamma \in \mathrm{SL}_{2}(\mathbb{Z})$ and for all $z \in \mathcal{H}$,

$$
F_{f}(\gamma(z))=J_{\gamma}(z) \rho_{l}(\gamma) \cdot F_{f}(z) .
$$

The dimension of the target space of $F_{f}$ depends on $l$ but not on the depth of $f$ if it is $<l$. The $D$-Wronskian

$$
W_{f}(z)=\operatorname{det}\left(F(z), D(F)(z), \ldots, D^{l-1}(F)(z)\right)
$$


is easily seen to be a modular form of weight $w(l+1)$ and, furthermore, we have $v\left(W\left(F_{f}\right)\right) \geq v(f)$. To fix the ideas of the construction, the reader can check the following formula by using (5):

$$
W\left(F_{E_{2}}\right)=-\frac{E_{4}}{2 \pi i} .
$$

If $f \in \widetilde{M}_{w}^{\leq l}$ is non-zero we have that $Q_{f}$ has degree $l$ in $X$ and the functions

$$
\left(\frac{\partial^{j} Q_{f}}{\partial x^{j}}\right)_{x=z}, \quad j=0, \ldots, l,
$$

are linearly independent over $\mathbb{C}$ so that $W\left(F_{f}\right) \in M_{(l+1) w} \backslash\{0\}$. We now look at $W\left(F_{f}\right)$ with $f=f_{l, w}$. By Lemma 2.1,

$$
\begin{aligned}
\delta_{l}(w)-1 & \geq d((l+1) w)-1-\kappa_{l} \\
& \geq v\left(W\left(F_{f_{l, w}}\right)\right)-\kappa_{l} \\
& \geq v_{\max }(l, w)-\kappa_{l} .
\end{aligned}
$$

If $l \in\{0, \ldots, 4\}$, then $\kappa_{l}=0$. Therefore, $v_{\max }(l, w)=\delta_{l}(w)-1$.

Proof of Theorem 1.3. We suppose that $l \in\{0,1,2,3,4\}$. Lemma 2.1 tells us that $\kappa_{l}(w)=$ $\kappa_{l}=0$ for all $w \in 2 \mathbb{Z}, w \geq 0$. Combining (3) and Theorem 2.3 we see that $v_{\max }(l, w)=$ $\delta_{l}(w)-1$. Hence an algebraically extremal quasi-modular form of weight $w$ and depth $l$ is also analytically extremal.

Let us consider $w \in 2 \mathbb{N}$. We have the flag of vector spaces

$$
M_{w} \subsetneq \tilde{M}_{w}^{\leq 1} \subsetneq \cdots \subsetneq \tilde{M}_{w}^{\leq w / 2-2} \subsetneq \tilde{M}_{w}^{\leq w / 2},
$$

which implies $v_{\max }(0, w)<v_{\max }(1, w)<\cdots<v_{\max }(w / 2-2, w)<v_{\max }(w / 2, w)$. This means that if $l \neq w / 2-1$, an analytically extremal quasi-modular form is algebraically extremal.

Remark 2.4. For $l \leq 4$ we have $\operatorname{dim}_{\mathbb{C}}\left(\tilde{M}_{w}^{\leq l}\right)=\operatorname{dim}_{\mathbb{C}}\left(M_{(l+1) w}\right)$ but we have not constructed natural isomorphisms $\widetilde{M}_{w}^{\leq l} \cong M_{(l+1) w}$. Hence, 'diagonal bases' exist for $l \leq 4$ but we have not provided a way to construct them explicitly. If $V:=\tilde{M}_{w}^{\leq l} \neq\{0\}$ the map $f \mapsto W\left(F_{f}\right)$ is homogeneous of weight $l+1$ over $V$ (for example, if $l=1$, it is quadratic). We consider its polarization

$$
V^{\oplus(l+1)} \stackrel{\Phi}{\rightarrow} M_{(l+1) w} .
$$

We address the following problem. Characterize the $l$-tuples $\left(f_{1}, \ldots, f_{l}\right) \in V^{\oplus l}$ such that the map $V \ni f \mapsto \Phi\left(f, f_{1}, \ldots, f_{l}\right) \in M_{(l+1) w}$ is an isomorphism of $\mathbb{C}$-vector spaces.

\section{Further remarks in depth one}

It belongs to Kaneko and Koike the discovery (in [5]) that (algebraically) extremal quasimodular forms of weight one and two are solutions of linear differential equations belonging to one-parameter families of hypergeometric type. One of the reasons for which the 
terminology 'hypergeometric' is used is that, moreover, the (algebraically) extremal forms (in depth one and two) can also be described inductively by using certain contiguity equations similar to those of Gauss' hypergeometric function first observed by Kaneko and Koike (see Lemma 3.7). The author noticed, in other works, that these contiguity equations can also be viewed as an avatar of an analytic family of Drinfeld modules of rank two.

From now on, we focus on the case of depth one and weight a multiple of six. We write

$$
\Delta=\frac{E_{4}^{3}-E_{6}^{2}}{1728} \in M_{12}
$$

and we set $D=D_{1}$. The following is a simple consequence of [5, Theorem 2.1 part (1)] observing that $D(\Delta)=E_{2} \Delta$ (which is clear from (5)). The symbol $\Delta^{1 / 2}$ denotes the unique normalized square root of $\Delta$ in $u \mathbb{C}[[q]]$, where $u:=q^{1 / 2}=e^{\pi i z}$. This can also be viewed as a holomorphic, nowhere-vanishing function over $\mathcal{H}$.

Proposition 3.1. If $w=6 k, k \in \mathbb{N}$, then the function $f_{1,6 k} \Delta^{-k / 2}$ is the unique solution in $u \mathbb{C}[[q]]$ of the differential equation

$$
D^{2}(X)=\frac{k^{2}}{4} E_{4} X
$$

Proof. First, we acknowledge that this is a direct consequence of the results of [5]. By [5, Theorem 2.1 part (1)] we have

$$
\left(D^{2}-\frac{w}{6} E_{2} D+\frac{w(w-1)}{12} D\left(E_{2}\right)\right)\left(f_{1, w}\right)=0, \quad w \in 6 \mathbb{N},
$$

and $f_{1, w}$ is the unique such solution in $\mathbb{Q}[[q]]$. Now, in the skew $\mathbb{C}$-algebra $\mathbb{C}\left[E_{2}, E_{4}, E_{6}, \Delta^{-1 / 2}\right][D]$ with $D c=c D+D(c), c \in \mathbb{C}\left[E_{2}, E_{4}, E_{6}, \Delta^{-1 / 2}\right]$, we have

$$
D^{2}-\frac{w}{6} E_{2} D+\frac{w(w-1)}{12} D\left(E_{2}\right)=\Delta^{k / 2}\left(D^{2}-\frac{k^{2}}{4} E_{4}\right) \Delta^{-k / 2},
$$

and this concludes the proof.

We can also proceed independently from [5, Theorem 2.1] by using Theorem 1.3 and some basic properties of Rankin-Cohen brackets in the following way. Note that $\delta_{1}(6 k)=\delta_{1}(6 k+4)=k+1$ for $k \in \mathbb{N}$. Hence, $v_{\max }(1,6 k)=v_{\max }(1,6 k+4)=k, k \in \mathbb{N}$. Now observe that

$$
g_{k}:=\left(\Delta^{k / 2}\left(D^{2}-\frac{k^{2}}{4} E_{4}\right) \Delta^{-k / 2}\right)\left(f_{1,6 k}\right)=\theta_{6 k-1}^{(1)}\left(f_{1,6 k}\right) \in \tilde{M}_{6 k+4}^{\leq 1}
$$

in the notation of [5, p. 467], thanks to [5, Proposition 3.3] (the proof of which uses the notion of $\mathfrak{s l}_{2}$-triple). Hence looking at $q$-expansions and using that $f_{1,6 k}=q^{k}+o\left(q^{k+1}\right)$ we see that $v\left(g_{k}\right)>v_{\max }(1,6 k+4)$ and $g_{k}=0$ for all $k$.

A normalized extremal quasi-modular form (of weight $w$ and depth $\leq l$ ) has its $q$ expansion which is defined over $\mathbb{Q}$; in other words, the coefficients of its $q$-expansion are rational numbers. By the fact that the $q$-expansions of $E_{2}, E_{4}$ and $E_{6}$ are rational integers, it is also clear that the denominators of these rational numbers are bounded in absolute value depending on $l$ and $w$. It is then natural to ask for various properties of these rational coefficients such as upper bounds for the primes dividing these denominators in the style of the Clausen-von Staudt theorem.

We recall [5, Conjecture 2, p. 469] the following. 
Conjecture 3.2. (Kaneko and Koike) If $l \in\{1,2,3,4\}$, then $f_{l, w}$ belongs to $\mathbb{Z}[1 / p: p<$ $w][[q]]$. Furthermore, except for $f_{1,2}=E_{2}$, the coefficients of the Fourier series of $f_{l, w}$ are all positive.

This supports Kaneko and Koike's prediction that these coefficients could be the values of some 'counting function of geometric nature'. In the direction of this conjecture, we have the next results.

THEOREM 3.3. For all $k \geq 0$ we have $f_{1,6 k} \in \mathbb{Z}[1 / p: p<6 k][[q]]$.

This result has also been independently noticed by Kaneko, along with the analogous statement for the case $l=2$ of the conjecture, as an application of the techniques introduced in [4] and [5]. We propose here to revisit these techniques, including the arguments of [5]. The novel observation is the use of certain identities of 'Lax type' (see Lemmas 3.4 and 3.5 below). We hope that these techniques can contribute to fully solve the conjecture.

We suppose that $k$ is now an indeterminate and we set $B:=\mathbb{Q}(k)\left[E_{2}, E_{4}, E_{6}, \Delta^{-1 / 2}\right]$. Note that $B$ embeds in the valued field $\mathbb{Q}(k)((u))$ where $u^{2}=q$. Also, $B$ and $\mathbb{Q}(k)((u))$ are endowed with the $\mathbb{Q}((u))$-linear automorphism $\sigma$ defined by $\sigma(k)=k+1$ and also with the $\mathbb{Q}(k)$-linear derivation induced by the system (5) and by $D(u)=u / 2$. These two $\mathbb{Q}$-vector space endomorphisms satisfy the commutation rules

$$
D \sigma=\sigma D, \quad D c=c D+D(c), \quad \sigma c=\sigma(c) \sigma, \quad c \in \mathbb{Q}(k)((u))
$$

and we can consider the skew polynomial Ore algebras

$$
B[D, \sigma] \subset \mathbb{Q}(k)((u))[D, \sigma],
$$

which can be identified with subalgebras of the $\mathbb{Q}$-linear endomorphisms of $B \subset \mathbb{Q}(k)((u))$. Let $\Psi$ be in $B[D, \sigma]$. Then, the map

$$
\partial_{\Psi}: B[D, \sigma] \rightarrow B[D, \sigma], \quad \partial_{\Psi}(x)=[x, \Psi]=x \Psi-\Psi x
$$

is a derivation. Also, $B[D, \sigma]$ is equipped with the $\mathbb{Q}\left[E_{2}, E_{4}, E_{6}, \Delta^{-1 / 2}\right]$-linear automorphism

$$
\Psi \mapsto \Psi^{(1)}:=\sigma \Psi \sigma^{-1} .
$$

In other words, $\Psi^{(1)} \in B[D, \sigma]$ is the unique element such that $\Psi^{(1)} \sigma=\sigma \Psi$ and, clearly, we can also define $\Psi^{(i)}$ for all $i \in \mathbb{Z}$. In addition, there is an obvious commutation rule between $X \mapsto X^{(1)}$ and $\delta_{\bullet}$. Similar properties as the above hold in the algebra $\mathbb{Q}(k)((u))[D, \sigma]$. We set

$$
E:=D^{2}-\frac{k^{2}}{4} E_{4} \in B[D, \sigma] .
$$

We are interested in the $\mathbb{Q}(k)$-vector space

$$
H:=\left\{\Psi \in B[D, \sigma]: \partial_{E}(\Psi) \in B[D, \sigma] E\right\},
$$

which is the intersection between the left ideal generated by $E$ and the image of $\partial_{E}$ in $B[D, \sigma]$. Clearly, $\mathbb{Q}(k) \subset H$. We set

$$
F:=\Delta^{-1 / 2}\left(E_{2} E_{4}+(5+6 k) E_{6}+12 E_{4} D\right) \sigma \in B[D, \sigma] .
$$


LEMMA 3.4. We have $\mathbb{Q}(k)[F] \subset H$.

Proof. It suffices to show that $F \in H$. This follows from an elementary computation which uses (5). More explicitly, we have the formula (our first identity of 'Lax type')

$$
\delta_{E}(F)=4 \Delta^{-1 / 2}\left(E_{2} E_{4}+2 E_{6}\right) \sigma E .
$$

Let $\mu(k)$ be an element of $\mathbb{Q}(k)$. We set

$$
G_{\mu}:=\sigma^{2}-\mu(k)\left(1-\frac{E_{6}}{\Delta^{1 / 2}} \sigma\right) \in B[\sigma] .
$$

LEMMA 3.5. The following identity holds:

$$
E^{(2)} G_{\mu}-G_{\mu} E=\mu(k) E_{4}\left(\frac{F}{12}-(k+1)\right) .
$$

Proof. This also follows from an elementary computation, independent this time from (5). Note that the identity holds regardless of the choice of $\mu(k)$.

The identity (9) is our second identity of 'Lax type'. We now proceed to construct formal solutions of (7). Let $Y$ be a formal solution of the equation $D(Y)=(k / 2) Y$ (in terms of analytic functions of two variables, we would have $\left.Y=e^{k z \pi i}\right)$. Since $\sigma$ and $D$ commute in $\mathbb{Q}(k)((u))[D, \sigma]$, we can give the field

$$
L=\mathbb{Q}(k)((u))(Y)
$$

the structure of a $\mathbb{Q}(k)((u))[D, \sigma]$-module by setting $\sigma(Y)=Y u$. If $f \in L$, and $\Psi \in$ $\mathbb{Q}(k)((u))[D, \sigma]$, we denote by $\Psi(f)$ the action of $\Psi$ on $f$ for this module structure (evaluation). It is easy to see that $Y$ and $Y^{-1}$ are linearly independent over $\mathbb{Q}(k)((u))$.

Let $\left(c_{n}(x)\right)_{n \geq 0}$ be the sequence of $\mathbb{Q}(x)$ with $c_{0}=1$, uniquely defined inductively as follows:

$$
c_{n}(x)=\frac{240 x^{2}}{n(n+2 x)} \sum_{i=1}^{n} \sigma_{3}(i) c_{n-i}(x) .
$$

This is just the recursive rule induced by (7) and we have

$$
\operatorname{Ker}(E)=\mathbb{Q}(k) \varphi_{1} \oplus \mathbb{Q}(k) \varphi_{2} \subset L,
$$

where

$$
\varphi_{1}=Y^{-1} \sum_{n \geq 0} c_{n}(-k / 2) q^{n}, \quad \varphi_{2}=Y \sum_{n \geq 0} c_{n}(k / 2) q^{n} .
$$

In particular, we have

$$
\varphi_{2}=Y\left(1+\frac{60 k^{2}}{k+1} q+o(q)\right) \in Y \mathbb{Q}(k)((q)) .
$$

It is also easy to see that $\varphi_{1}$ and $\varphi_{2}$ are linearly independent over $\mathbb{Q}(k)((u))$. 
LEMMA 3.6. We have

$$
G_{\mu}(\varphi)=Y o(1)
$$

in $Y \mathbb{Q}(k)((q))$ if and only if

$$
\mu(k)=\frac{(1+k)(2+k)}{12(7+6 k)(11+6 k)} .
$$

Proof. This is straightforward using (11). We have

$$
\sigma^{2}\left(\varphi_{2}\right)=q Y\left(1+\frac{60(k+2)^{2}}{k+3} q+o(q)\right) .
$$

Moreover, we have, by the expansion $\Delta^{-1 / 2}=u^{-1}+12 u+o\left(u^{3}\right)$ (which easily follows from, say, the product formula of $\Delta$ ),

$$
\mu(k)\left(1-\frac{E_{6}}{\Delta^{1 / 2}} \sigma\right)\left(\varphi_{2}\right)=\mu(k) Y\left(-\frac{12(7+6 k)(11+6 k)}{(1+k)(2+k)} q+o(q)\right),
$$

and the result follows.

From now on we set $\mu(k)$ as in (12) and $G=G_{\mu}$.

LEMMA 3.7. We have $G\left(\varphi_{2}\right)=0$.

Proof. We set $V:=\operatorname{Ker}(E) \cap Y \mathbb{Q}(k)((u))$; this is a subvector space over $\mathbb{Q}(k)$ of $L$. Note that $\varphi:=\varphi_{2} \in V$ and that $\varphi_{1} \notin V$. Indeed, otherwise we would have $\varphi_{1} \in Y \mathbb{Q}(k)((u))$, contradicting the above-mentioned property that $Y$ and $Y^{-1}$ are linearly independent over $\mathbb{Q}(k)((u))$. Hence $V$ is one-dimensional, generated by $\varphi$. By Lemma 3.4, $F$ determines an endomorphism of $V=\mathbb{Q}(k) \varphi$ and therefore $\varphi$ is an eigenvector of $F$ with eigenvalue $\lambda \in \mathbb{Q}(k)$. By using (10) this can be easily computed:

$$
\lambda=k+1 .
$$

By Lemma 3.5, $E^{(2)}(G(\varphi))=0$ and we immediately see that $\sigma^{-2}(G(\varphi)) \in \operatorname{Ker}(E)$. Hence, $G(\varphi) \in \mathbb{Q}(k) \sigma^{2}(\varphi)$. We write $G(\varphi)=\eta \sigma^{2}(\varphi)$ with $\eta \in \mathbb{Q}(k)$. By Lemma 3.6, $\eta=0$.

Proof of Theorem 3.3. By using (10) and induction, we see that for all $i, j \geq 0, \sigma^{i}\left(c_{j}(k / 2)\right) \in$ $\mathbb{Q}(k)$ is regular at $k=0$. Hence, by Proposition 3.1, for all integers $i \geq 0$, writing

$$
\left.\sigma^{i}\left(\varphi_{2}\right)\right|_{k=0}=u^{i} \sum_{j \geq 0} c_{i, j} q^{j},
$$

we have

$$
f_{1,6 i} \Delta^{-i / 2}=u^{i} \sum_{i \geq 0} c_{i, j} q^{j}
$$

To finish our proof, all we need to show is that, if $p$ is a prime number dividing the denominator of $c_{i, j}$, then $p<6 i$. The property is obvious for $i=0$; indeed, one immediately sees that $\left.\varphi_{2}\right|_{k=0}=1$. For $i=1$ we know that $f_{1,6}=\left(E_{2} E_{4}-E_{6}\right) / 720$ has $q$-expansion defined over $\mathbb{Z}\left[\frac{1}{2}, \frac{1}{3}, \frac{1}{5}\right]$ because $720=2^{4} \cdot 3^{2} \cdot 5$. Therefore $\left.\left(\sigma\left(\varphi_{2}\right)\right)\right|_{k=0}=f_{1,6} \Delta^{-1 / 2}$ has the required property (notice that $\Delta^{1 / 2}$ is defined over $\mathbb{Z}$ ). 
We now suppose, by induction hypothesis, that for all $i^{\prime}<i$, if $p$ divides the denominator of $c_{i^{\prime}, j}$ for some $j$, then $p<6 i^{\prime}$. Since the $q$-expansion of $E_{6} \Delta^{-1 / 2}$ is defined over $\mathbb{Z}$ and does not depend on $k$, we have that the $q$-expansion $u^{i} \sum_{j \geq 0} r_{j} q^{j}$ of $\left(\sigma^{i-2}\left(\varphi_{2}\right)-\right.$ $\left.E_{6} \Delta^{-1 / 2} \sigma^{i-1}\left(\varphi_{2}\right)\right)\left.\right|_{k=0}$ is well defined and has rational coefficients whose primes dividing the denominators do not exceed $6 i-6$. But now,

$$
c_{i, j}=\frac{i(i-1)}{12(6 i-1)(6 i-5)} r_{j},
$$

which implies that if $p$ is a prime dividing the denominator of $c_{i, j}$, then $p<6 i$. Notice that the argument must be slightly modified if $p=2$ or 3 . The proof of the theorem now follows easily by the fact that $f_{1,6 i}=\left.\Delta^{i / 2}\left(\sigma^{i}\left(\varphi_{2}\right)\right)\right|_{k=0}$, because, as previously noticed, the $q$-expansion of $\Delta^{1 / 2}$ is defined over $\mathbb{Z}$.

Remark 3.8. We have been unable to show that $f_{1,6 i}$ has positive Fourier coefficients for $i \geq 0$. However, from (10), it is easy to deduce that the Fourier coefficients of $f_{1,6 i} \Delta^{-i / 2}$ are non-negative, for all $i \geq 0$.

We conclude the paper with a prediction. In [5], several examples are given, providing experimental evidence of the truth of Conjecture 3.2. We conducted similar numerical examples and we noticed that many normalized extremal quasi-modular forms $f_{l, w}$ with $l \leq 4$ seem to have integral Fourier coefficients. It is also apparent that this phenomenon ceases to hold for $l>4$. Let $\mathcal{E}_{l}$ be the set of integers $w$ such that $f_{l, w} \in \mathbb{Z}[[q]]$. We propose the following, based on numerical investigations we did.

CONJECture 3.9. If $l \in\{1,2,3,4\}$, then $\mathcal{E}_{l}$ is an infinite set. If $l>4$, then $\mathcal{E}_{l}$ is a finite set.

If $l>4$, we did not find any candidate for an element of $\mathcal{E}_{l}$.

\section{A. Appendix by G. Nebe: an example}

In 'small' weight, it is easy to show that an extremal quasi-modular form of depth one has positive integral coefficients. For example, we have used that

$$
f_{1,6}=\frac{D\left(E_{4}\right)}{240} \in \mathbb{Z}\left[\frac{1}{2}, \frac{1}{3}, \frac{1}{5}\right][[q]]
$$

(and the coefficients are positive) but, even better, this series is in $\mathbb{Z}[[q]]$ by (1). Apart from this and other simple examples, it is not easy to construct extremal quasi-modular forms in $\mathbb{Z}[[q]]$ with non-negative coefficients. As Kaneko and Koike pointed out in [5], the normalized extremal quasi-modular form of weight six and depth three,

$$
f_{3,6}=\sum_{i \geq 2} c_{i} q^{i}
$$

(with $c_{2}=1$ ), has the following property. The coefficient $c_{d}$ is equal to the number of simply ramified coverings of genus two and degree $d$ of an elliptic curve over $\mathbb{C}$, hence providing another example of a normalized extremal quasi-modular form with positive integral coefficients (see $[2,6])$.

In this appendix we show that these properties are also shared with the normalized extremal form $f_{1,14}$. Let us consider the theta series (of weight 12) associated to the Leech 
lattice $\Lambda_{24}$ :

$$
\theta_{\Lambda_{24}}=E_{12}-\frac{65520}{691} \Delta
$$

where $E_{12}$ denotes the normalized Eisenstein series of weight 12 . We have $\theta_{\Lambda_{24}} \in \mathbb{Z}[[q]]$ and the coefficients are non-negative. By using the well-known fact that $\theta_{\Lambda_{24}}$ is an extremal modular form of weight 12 and that $v_{\max }(1,14)=2$ (by Theorem 1.3 or by numerical computations), we easily deduce that

$$
f_{1,14}=\frac{1}{393120} D\left(\theta_{\Lambda_{24}}\right) .
$$

Here,

$$
393120=2^{5} \cdot 3^{3} \cdot 5 \cdot 7 \cdot 13
$$

is twice the number of minimal vectors in $\Lambda_{24}$ and we see that $f_{1,14} \in \mathbb{Z}\left[\frac{1}{2}, \frac{1}{3}, \frac{1}{5}, \frac{1}{7}, \frac{1}{13}\right][[q]]$ while the coefficients of the Fourier series are all non-negative, in agreement with Conjecture 3.2. But more is true.

Theorem A.1. We have $f_{1,14} \in \mathbb{Z}[[q]]$.

Proof. To prove the theorem, let $L:=\Lambda_{24}$ be the Leech lattice and $A:=393120=2^{5} \cdot 3^{3}$. $5 \cdot 7 \cdot 13$. By the above

$$
f_{1,14}=A^{-1} q \sum_{a=1}^{\infty} a\left|L_{a}\right| q^{a} \quad \text { where } L_{a}=\left\{\lambda \in L \mid\|\lambda\|^{2}=a\right\} .
$$

To show the theorem, we need to show that $A$ divides $a\left|L_{a}\right|$ for all $a$. We do this prime by prime.

Let $G$ be the automorphism group of $L$. Then $G \cong 2 . C o_{1}$ has order $|G|=2^{22} 3^{9} 5^{4} 7^{2} 11$. $13 \cdot 23$ and acts on the finite set $L_{a}$ for all $a>0$. For a subgroup $S \leq G$ and $\lambda \in L_{a}$ we put

$$
S \cdot \lambda:=\{\sigma(\lambda) \mid \sigma \in S\}
$$

to denote the orbit of $\lambda$ under $S$. Then $L_{a}$ is a disjoint union of $S$-orbits and, by the Lagrange theorem, $|S \cdot \lambda|=|S| /|U|$ where $U=\{\sigma \in S \mid \sigma(\lambda)=\lambda\}$ is the stabilizer in $S$ of $\lambda$.

To see that $5 \cdot 7 \cdot 13$ divides $\left|L_{a}\right|$ let $p \in\{5,7,13\}$. Then the Sylow- $p$-subgroup $S$ of $G$ acts fixed point freely on the non-zero vectors in $L$. So the stabilizer $U$ of any $\lambda \in L_{a}$ has index $>1$ and all $S$-orbits in $L_{a}$ have length a multiple of $p$.

Now let $S$ be a Sylow-3-subgroup of $G$, so $S \leq G$ has order $3^{9}$. With Magma [1] we computed the low-index subgroups of $S$ of index one, three and nine. For all 101 of these 102 subgroups $U$, the sublattice of $L$ consisting of all elements of $L$ that are fixed by all generators of $U$ is $\{0\}$. For the other subgroup $U$ (of index nine in $S$ ) this sublattice is isometric to ${ }^{(3)} A_{2}$. In particular, all elements in the lattice have norm divisible by 3 . So whenever there is $\lambda \in L_{a}$ such that $|S \cdot \lambda| \leq 9$, then $|S \cdot \lambda|=9$ and $\lambda$ lies in a sublattice of $L$ that is isometric to ${ }^{(3)} A_{2}$. In particular, $a$ is a multiple of 3 . This shows that 9 divides $\left|L_{a}\right|$ and $3^{3}$ divides $a\left|L_{a}\right|$ for all $a>0$.

To see the divisibility by $2^{5}$, we could argue the same way, but Magma failed to compute the low-index subgroups of the Sylow-2-subgroup (of order $2^{22}$ ) of $G$ up to index 16. Instead, 
let $\sigma \in G$ be an element of order 16 (it is not unique, but the following holds for all three conjugacy classes). Let

$$
K:=\left\{\lambda \in L \mid \sigma^{8}(\lambda)=\lambda\right\} \quad \text { and } \quad N:=\left\{\lambda \in L \mid \sigma^{8}(\lambda)=-\lambda\right\} .
$$

We compute that $K={ }^{(2)} E_{8}$ is the rescaled Barnes-Wall lattice of dimension eight and $N \cong \Lambda_{16}$ is the Barnes-Wall lattice of dimension 16. By [3, Theorem 14] all non-zero shells $K_{a}(a>0)$ have a cardinality divisible by $2 \cdot 8=2^{4}$ and similarly $2 \cdot 16=2^{5}$ divides $\left|N_{a}\right|$ for all $a>0$. Note also that $K_{a}=\emptyset$, if $a$ is odd.

Let $M_{a}:=L_{a} \backslash\left(K_{a} \cup N_{a}\right)$. Then

$$
M_{a}=\left\{\lambda \in L_{a}||\langle\sigma\rangle \cdot \lambda \mid=16 \text { and }-\lambda \notin\langle\sigma\rangle \cdot \lambda\right\}
$$

is a disjoint union of an even number of $\langle\sigma\rangle$-orbits of length 16. In particular $M_{a}$ has a cardinality divisible by $2 \cdot 16=2^{5}$. By the above $\left|N_{a}\right|$ is a multiple of $2^{5}$ and $K_{a}$ is either empty or $a$ is even and $\left|K_{a}\right|$ is a multiple of $2^{4}$. In total we have $a\left|L_{a}\right|=a\left|M_{a}\right|+a\left|N_{a}\right|+$ $a\left|K_{a}\right|$ is a multiple of $2^{5}$.

Note that the divisibility by $2^{5} 3^{3}$ of the coefficients of $D\left(\Theta_{\Lambda_{24}}\right)$ also follows from [7, Theorems 1.2 and 1.3].

\section{REFERENCES}

[1] W. Bosma, J. Cannon and C. Playoust. The Magma algebra system. I. The user language. J. Symbolic Comput. 24 (1997) 235-265.

[2] R. Dijkgraaf. Mirror symmetry and elliptic curves. The Moduli Space of Curves (Progress in Mathematics, 129). Birkhäuser, 1995, pp. 149-163.

[3] N. Heninger, E. M. Rains and N. J. A. Sloane. On the integrality of $n$th roots of generating functions. J. Combin. Theory Ser. A 113 (2006) 1732-1745.

[4] M. Kaneko and M. Koike. On modular forms arising from a differential equation of hypergeometric type. Ramanujan J. 7 (2003) 145-164.

[5] M. Kaneko and M. Koike. On extremal quasimodular forms. Kyushu J. Math. 60 (2006) 457-470.

[6] M. Kaneko and D. Zagier. A generalized Jacobi theta function and quasimodular forms. The Moduli Space of Curves (Progress in Mathematics, 129). Birkhäuser, 1995, pp. 165-172.

[7] M. Koike. Congruences between extremal modular forms and theta series of special types modulo powers of 2 and 3. Kyushu J. Math. 63 (2009) 123-132.

\section{Federico Pellarin \\ Institut Camille Jordan \\ UMR 5208 \\ Site de Saint-Etienne}

23 rue du Dr. P. Michelon

42023 Saint-Etienne

France

(E-mail:federico.pellarin@univ-st-etienne.fr)

\author{
Gabriele Nebe \\ Lehrstuhl für Algebra und Zahlentheorie \\ RWTH Aachen University \\ 52056 Aachen \\ Germany \\ (E-mail: gabriele.nebe@rwth-aachen.de)
}

